\title{
High-mobility group A1 inhibits p53 by cytoplasmic relocalization of its proapoptotic activator HIPK2
}

\author{
Giovanna Maria Pierantoni, ${ }^{1}$ Cinzia Rinaldo, ${ }^{2}$ Marcella Mottolese, ${ }^{3}$ Anna Di Benedetto, ${ }^{3}$ \\ Francesco Esposito, ${ }^{1}$ Silvia Soddu, ${ }^{2}$ and Alfredo Fusco ${ }^{1,4}$
}

\begin{abstract}
1Dipartimento di Biologia e Patologia Cellulare e Molecolare, Facoltà di Medicina e Chirurgia, Università degli Studi di Napoli "Federico II," Naples, Italy. 2Dipartimento di Oncologia Sperimentale and "Servizio di Anatomia Patologica, Istituto Nazionale dei Tumori Regina Elena, Rome, Italy. ${ }^{4}$ Naples Oncogenomic Center-Centro di Ingegneria Genetica (NOGEC-CEINGE), Biotecnologie Avanzate, and Scuola Europea di Medicina Molecolare (SEMM), Naples, Italy.
\end{abstract}

\begin{abstract}
High-mobility group A1 (HMGA1) overexpression and gene rearrangement are frequent events in human cancer, but the molecular basis of HMGA1 oncogenic activity remains unclear. Here we describe a mechanism through which HMGA1 inhibits $\mathrm{p} 53$-mediated apoptosis by counteracting the $\mathrm{p} 53$ proapoptotic activator homeodomain-interacting protein kinase 2 (HIPK2). We found that HMGA1 overexpression promoted HIPK2 relocalization in the cytoplasm and inhibition of $\mathrm{p} 53$ apoptotic function, while HIPK2 overexpression reestablished HIPK2 nuclear localization and sensitivity to apoptosis. HIPK2 depletion by RNA interference suppressed the antiapoptotic effect of HMGA1, which indicates that HIPK2 is the target required for HMGA1 to repress the apoptotic activity of p53. Consistent with this process, a strong correlation among HMGA1 overexpression, HIPK2 cytoplasmic localization, and low spontaneous apoptosis index (comparable to that observed in mutant p53-carrying tumors) was observed in WT p53-expressing human breast carcinomas. Hence, cytoplasmic relocalization of HIPK2 induced by HMGA1 overexpression is a mechanism of inactivation of p53 apoptotic function that we believe to be novel.
\end{abstract}

\section{Introduction}

The high-mobility group (HMG) proteins are low-molecular weight nuclear factors with nonhistone chromosomal accessory functions (1). The A subgroup of HMG (i.e., HMGA1a, HMGA1b, HMGA1c, and HMGA2) interacts with the minor groove of many AT-rich promoters and enhancers (2) and plays key roles in chromatin architecture and gene transcription control $(2,3)$. HMGA proteins do not directly exert transcriptional activity and are considered architectural transcription factors. By a complex network of protein-DNA and protein-protein interactions, they organize chromatin into the structure required by the basal transcription machinery to execute gene transcription (4). In physiological conditions, HMGA proteins are expressed at a high level during embryogenesis $(5,6)$, while their expression becomes low to undetectable in adult tissues. High HMGA expression in adult life is associated only with pathological conditions such as human carcinomas of thyroid $(7,8)$, colon (9-11), prostate (12), pancreas (13), cervix (14), ovary (15), and breast (16) tissues. A functional role of this aberrant HMGA overexpression in cancers has been previously demonstrated. We previously showed that in rat thyroid cells, blockage of HMGA synthesis prevents tumor transformation by murine transforming retroviruses $(17,18)$. Furthermore, infection of different carcinoma cells by a recombinant adenovirus carrying the HMGA1b cDNA in antisense orientation induces

Nonstandard abbreviations used: Ad, adenovirus; EGFP, enhanced GFP; HIPK2, homeodomain-interacting protein kinase 2; HMG, high-mobility group; LMB, leptomycin B; MDM2, mouse double minute 2; NF-YB, nuclear factor YB; TCE, total cell extract.

Conflict of interest: The authors have declared that no conflict of interest exists. Citation for this article: J. Clin. Invest. 117:693-702 (2007). doi:10.1172/JCI29852. apoptosis and interferes with tumor growth in vivo (19). To get clues on the mechanism(s) through which HMGA proteins exert their tumorigenic effect, we recently searched for HMGA interactors by analysis of $\mathrm{Ab}$ arrays and found that HMGA1 binds the p53 oncosuppressor and decreases its apoptotic activity, strongly supporting the idea that HMGA1 contributes to tumor transformation by interfering with the apoptotic function of p53 (20). HMGA1 exerts this effect by repressing the p 53 transcriptional activity on target genes such as $B A X$ and $p 21^{\text {Waf }}$, and by chromatin reimmunoprecipitation experiments we showed that HMGA1 and p53 are present in the same complexes bound to the $B A X$ gene promoter. However, despite the negative action of HMGA1 on p53 transcriptional activity on this promoter, HMGA1 overexpression did not decrease the presence of p53 in this region, suggesting a mechanism of $\mathrm{p} 53$ inactivation independent of its DNA-binding activity, at least on this promoter.

The WT p53 protein, which is mutated or inactivated in most human cancers, is a master regulator of the apoptotic program $(21,22)$. p53 is a sequence-specific transcription factor that regulates the expression of genes involved in cell cycle arrest or apoptosis in response to genotoxic damage or cell stress, including tumor cell response to many antineoplastic treatments (23). It has been proposed that the posttranslational modifications that activate p53 are responsible for p53-mediated biological outcomes $(24,25)$. Among the $\mathrm{p} 53$ posttranslational modifications, phosphorylation at serine residue 46 (Ser46) was identified as a specific modification involved in apoptosis, at least in part through transcriptional regulation of specific target genes (26-28). We and others have previously shown that homeodomain-interacting protein kinase 2 (HIPK2) binds to and activates $\mathrm{p} 53$ by phosphorylating it at Ser 46 $(29,30)$. Furthermore, HIPK2 was also shown to promote apopto- 

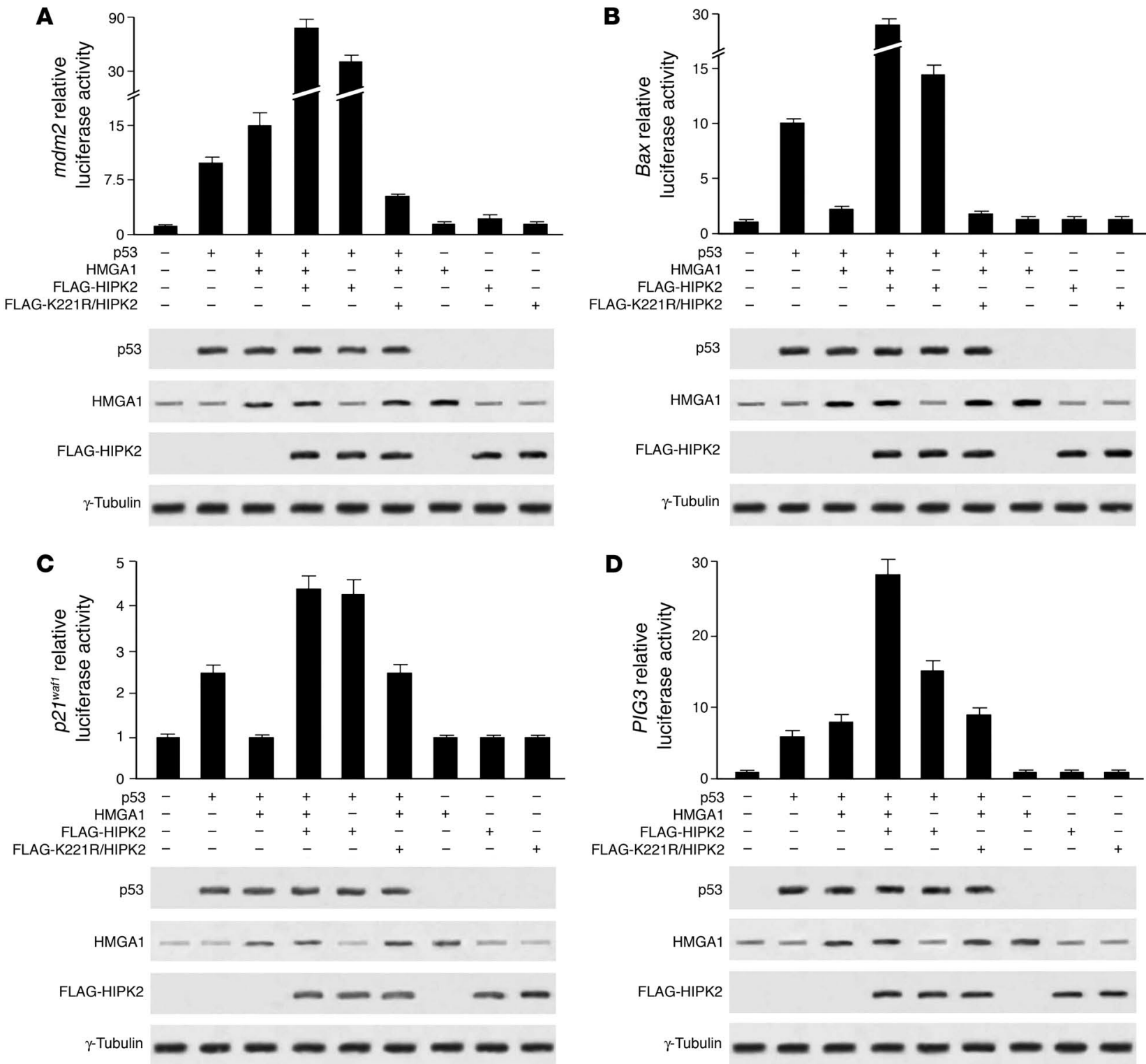

Figure 1

HMGA1, p53, and HIPK2 coexpression activates proapoptotic genes. Effect of FLAG-tagged WT HIPK2 or the kinase-defective HIPK2 mutant (FLAG-K221R/HIPK2) on the activity exerted by HMGA1 on reporter vectors mdm2 (A), bax (B), p21waf1 (C), and PIG3 (D). p53-null H1299 cells were used as recipient. The pCMV-p53 vector encoding WT p53 protein was transfected alone or with the indicated plasmids. All transfections were performed in duplicate; data are mean \pm SD of 5 independent experiments. Empty vectors were used as a control. Western blot analyses of p53, HMGA1, and FLAG-HIPK2 proteins expression from 1 indicative experiment are shown in the lower panels.

sis by acting on targets different from p53, such as the p53 family members p73 and p63 (31), the transcriptional corepressor c-terminal binding protein (32), the p53 inhibitor mouse double minute 2 (MDM2) (33), and the scaffold protein Axin (34), supporting a major role for HIPK 2 in the regulation of apoptosis. In addition, we showed that HIPK2 physically interacts with HMGA1b in vitro and in vivo, but the HIPK2-mediated function apparently occurs regardless of this interaction (35).

Here we report that HMGA1 interferes with p53-induced apoptosis by counteracting its proapoptotic activator HIPK2. In particular, HMGA1 overexpression prevented the nuclear localization of HIPK2 and the phosphorylation of p53Ser46, while these events were restored by HIPK2 overexpression or HMGA1 downregulation by tumor cell treatment with HMGA1-specific antisense oligonucleotides. Consistent with a causal role for HIPK2 relocalization in the HMGA1-induced inhibition of p53 apoptotic activity, HIPK2 depletion by RNA interference suppressed the antiapoptotic effect of HMGA1 expression. In agreement with these data, immunohistochemical analyses of biopsy tissue from 69 human breast carcinomas showed significant association among HMGA1 overexpression, HIPK2 cytoplasmic localization, and low spontaneous apoptosis index in the presence of WT p53. Our data support the hypothesis that HMGA1 exerts its tumorigenic activity by forcing a cytoplasmic localiza- 
tion of the p53 proapoptotic activator HIPK2, thus inhibiting p53 apoptotic function.

\section{Results}

HIPK2 is involved in the HMGA1/p53-mediated transcriptional regulation of p53 target genes. Results of our recent studies indicate that HMGA1 interferes with the p53-mediated transcription of the p53 effectors p $21^{\text {waf } 1}$ and Bax while cooperating with p53 in the transcription of its inhibitor MDM2. We did not observe any detectable modification in the amount of $\mathrm{p} 53$ present on the $B A X$ promoter analyzed by chromatin reimmunoprecipitation experiments upon HMGA1 overexpression (20). Thus, we asked whether other nuclear factors are responsible for the HMGA1 effects on p53 transcription. We concentrated our attention on HIPK2 for 2 main reasons: (a) HIPK2 contributes to p53 promoter selection by direct p53 phosphorylation at Ser46 and indirect p53 acetylation (28-30, 36, 37) and (b) HIPK2 binds HMGA1, and the functional role of this interaction is unknown (35). To evaluate whether HIPK2 expression affects HMGA1/p53-mediated transcription, we measured the activity of $\mathrm{p} 53$-dependent promoters in the presence of various combinations of p53, HMGA1, and HIPK2 proteins. p53-null H1299 cells were cotransfected with expression vectors encoding HMGA1 and p53 in combination or not with HIPK2 and with reporter vectors carrying the luciferase gene under the control of $\mathrm{p} 53$-responsive promoters $m d m 2, B A X$, $p 21^{W A F 1}$, and PIG3. As expected from our recent observations (20), HMGA1 cooperated with p53 in the transcription of its inhibitor MDM2 (Figure 1A), while it repressed the p53mediated transcription of the p53 effectors $B A X$ and $p 21^{\text {WAFI }}$ (Figure 1, B and C). No effect was observed on the PIG3 promoter (Figure 1D). This HMGA1 activity was dependent on the presence of p53, because the sole expression of HMGA1 did not affect promoter activities (Figure 1). To test whether HIPK2 contributes to this transcriptional regulation, we coexpressed HIPK2 with p53 and HMGA1. HIPK2 dramatically increased the activation of the $m d m 2$ and PIG3 promoters, but reverted the repressive effect exerted by HMGA1 on the $B A X$ and $p 21^{\text {WAF1 }}$ promoters (Figure 1). Indeed, except for the $p 21^{\text {WAF1 }}$ promoter, the activation induced by coexpression of the 3 factors exceeded that induced by HIPK2 and p53 without HMGA1. This effect can be attributed to HIPK2 activity, because the kinase-dead HIPK2-K221R mutant did not affect or even rescue the HMGA1/p53 transcriptional activity. Taken together, these results indicate that HIPK2 reverts the inhibitory activity of HMGA1 on the p53 effector promoters and points to a complex interplay among p53, HIPK2, and HMGA1 in the regulation of p53-target gene expression.

HIPK2 reverts the effects of HMGA1 on $p 53$ apoptotic function. We previously showed that the variation of p53-target gene transcription induced by HMGA1 overexpression was associated with reduction of p53 apoptotic activity (20). Because HIPK2 is a strong proapoptotic activator of p53 and its coexpression with HMGA1 modifies the HMGA1 activity on p53-mediated transcription, we examined whether HIPK2 counteracts the antiapoptotic effect of HMGA1. p53-null H1299 cells were transiently transfected with expression vectors encoding for HMGA1 or HIPK2, alone or in combination, and infected with a WT p53 recombinant adenovirus (Adp53) or an empty control virus (dl70.3). Expressions of the transduced proteins were determined by Western blot analyses (Figure 2A, lower panel). Cell death was measured by trypan blue exclusion (Figure 2A, upper panel) and TUNEL assay (Figure 2B), which revealed that HIPK2 overexpression inhibited the antiapoptotic activity of HMGA1 and induced a level of cell death comparable to that promoted by $\mathrm{p} 53 / \mathrm{HIPK} 2$ coexpression. In order to evaluate whether these results can be reproduced in more physiological conditions such as in WT p53-carrying cells during cell stress, human colon carcinoma RKO cells were induced to either over- or underexpress HMGA1 by transfection of HA-HMGA1encoding vector or HMGA1-specific antisense oligonucleotide.
A
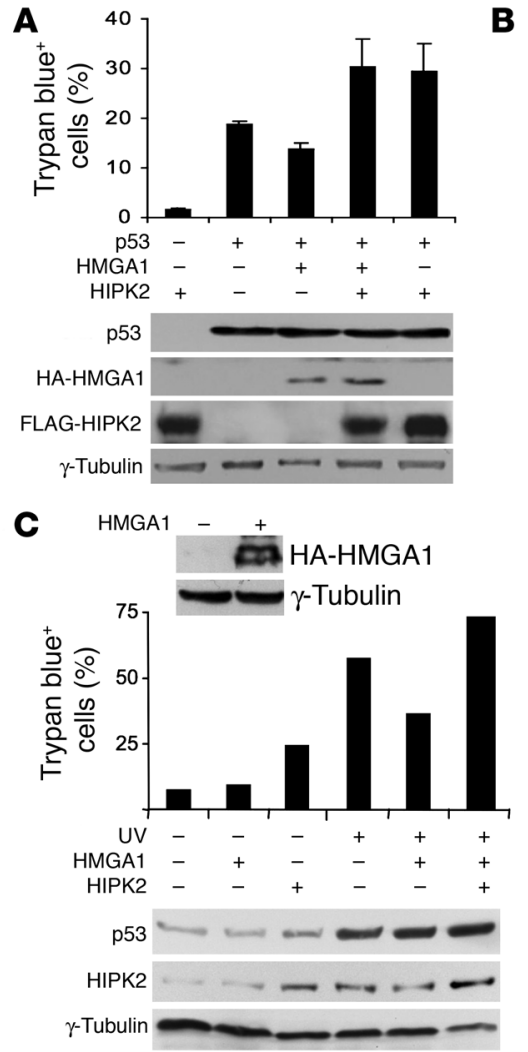

B

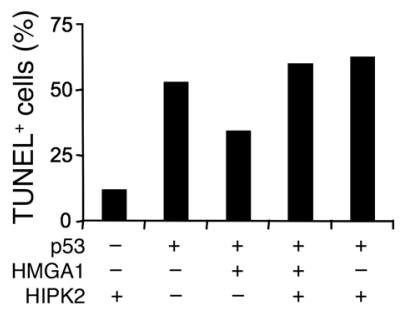

Figure 2

HIPK2 rescues the HMGA1 antiapoptotic effect on p53. (A) H1299 cells were infected with dl70.3 ( - in the p53 row) or Adp53 (+ in the p53 row) viruses at $60 \mathrm{MOI}$. One hour after infection, cells were transfected with pCEFL-Hmga1 vector or pCEFL control vector and pEGFP-HIPK2 vector or $\mathrm{pEGFP}$ empty vector. After 48 hours, floating and adherent cells were collected and analyzed by trypan blue exclusion. Mean \pm SD of 3 independent experiments are shown. Expression of the indicated proteins was analyzed by Western blot; the results of 1 indicative experiment of the 3 performed are reported. Expression of $\gamma$-tubulin shows equal loading of samples. (B) The same cells reported in A were analyzed by TUNEL assay. One indicative experiment is reported. (C) WT p53-carrying RKO cells overexpressing HA-HMGA1 protein or not, as detected by Western blot (upper panel), were transiently transfected with HIPK2 expression vector as indicated and subjected or not to UV light irradiation $\left(50 \mathrm{~J} / \mathrm{m}^{2}\right)$, and the percentage of cell death was measured by trypan blue exclusion 36 hours after irradiation. Expression of the indicated proteins was analyzed by Western blot; shown is 1 indicative experiment of the 3 performed. (D) RKO cells were transiently transfected with HMGA1-specific sense (S) or antisense (AS) oligonucleotides to reduce HMGA1 expression, as detected by Western blot (upper panel). Upon transfection of HIPK2 expression vector, cells were irradiated with UV light and analyzed as in C. 


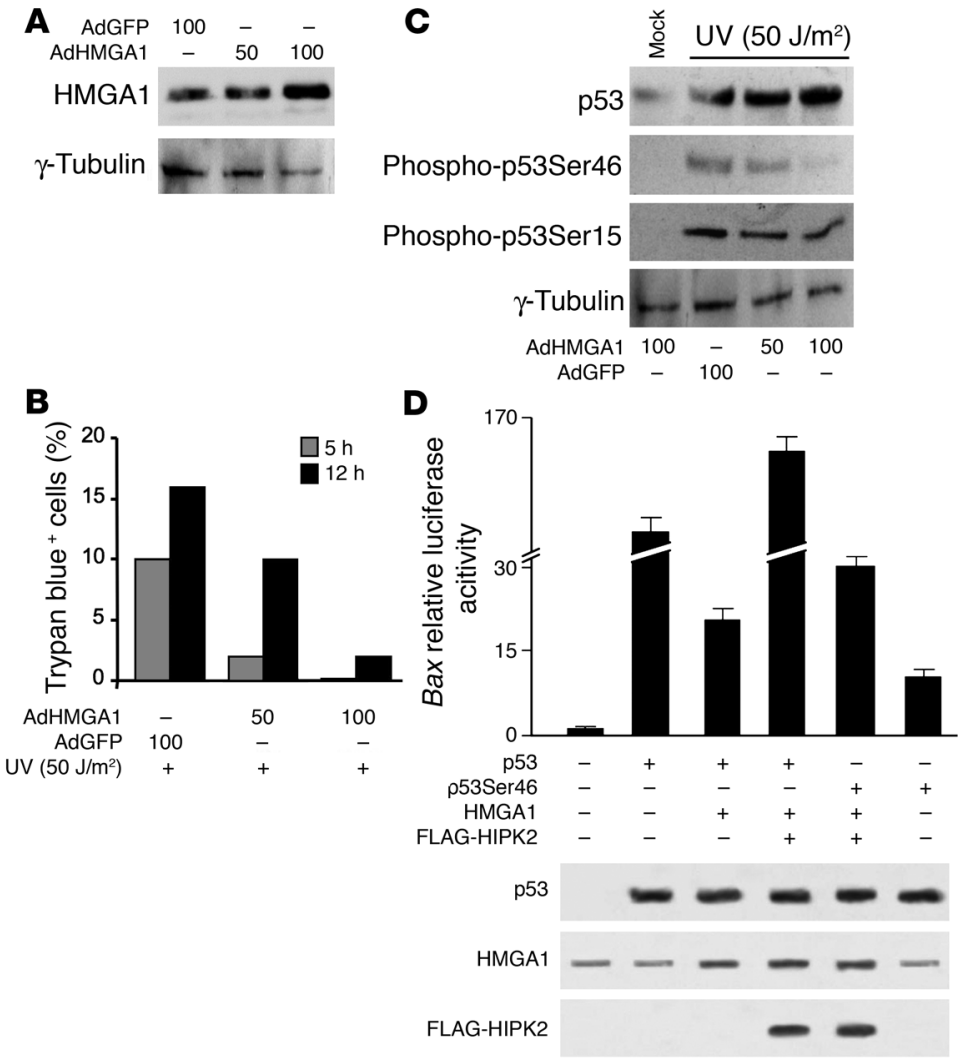

Figure 3

Contribution of p53 phosphorylation at Ser46 to HMGA1/HIPK2-induced BAX promoter activity. (A) Western blot analysis of the indicated protein in WT p53carrying HCT116 cells 48 hours after infection at the indicted MOI. (B) The same cells analyzed in A were treated with UV light at $50 \mathrm{~J} / \mathrm{m}^{2}$. At 5 and 12 hours after UV light irradiation, the rate of cell death was measured by trypan blue exclusion. (C) The same cells treated and analyzed in $\mathbf{B}$ were harvested at 5 hours after UV light irradiation to perform Western blot of the indicated proteins. (D) p53-null H1299 cells were transfected with the indicated expression vectors, and luciferase activity was determined. All transfections were performed in duplicate; data are mean \pm SD of 3 independent experiments. Empty vectors served as control. The expression of p53, HMGA1, and HIPK2 proteins was determined by Western blot.

Activation of endogenous p53 by UV light was associated with induction of cell death that, as expected from our previous results (20), was partially inhibited by HMGA1 overexpression. Consistent with the results obtained above by exogenous $\mathrm{p} 53$ expression, HMGA1-induced inhibition of cell death was completely overcome by HIPK2 (Figure 2C). In addition, in RKO cells transfected with HMGA1-specific sense or antisense oligonucleotides, HIPK2 overexpression increased cell susceptibility to UV light as well as HMGA1 depletion, and both HMGA1 depletion and HIPK2 overexpression further induced cell death (Figure 2D). Taken together, these results suggest that HIPK2 might be at least one of the targets for the antiapoptotic activity of HMGA1.

Phosphorylation of $p 53$ at Ser46 is involved in the antiapoptotic activity of HMGA1 overexpression. Phosphorylation of human p53 at Ser46 determines promoter selection and induction of apoptosis (27, 28). Because HIPK2 phosphorylates p53 at Ser46 and the kinasedead HIPK2-K221R mutant did not modify the p53-mediated transcriptional activity, we asked whether p53Ser46 phosphory- lation is involved in the HMGA1-mediated inhibition of p53 activity. The WT p53-carrying HCT116 cells were infected with WT HMGA1 recombinant adenovirus (AdHMGA1) or control virus (AdGFP) at the same MOI (Figure 3A) and treated or not with an apoptotic dose of UV light. As expected from our previous data (Figure 2C and ref. 20), HMGA1 overexpression protected cells from apoptosis in a dose-dependent manner (Figure 3B). Interestingly, this protection was associated with reduced phosphorylation of p53Ser46 (Figure 3C). Comparable results were obtained in RKO cells (data not shown). No difference was observed in the total amount of p53 or in its phosphorylation at Ser15, a HIPK2-independent posttranslational modification involved in p53 activation for either growth arrest or apoptotic functions $(24,25)$, which supported a direct role for the inhibition of the p53Ser46 phosphorylation pathway in apoptosis resistance induced by HMGA1 overexpression.

Next, we measured HMGA1/HIPK2-mediated transcription of the $B A X$ promoter in the presence of the $\mathrm{p} 53 \mathrm{Ser} 46 \mathrm{~A}$ mutant, which cannot be phosphorylated by HIPK2. The p53Ser46A mutant was much less efficient than WT p53 in activating the $B A X$ promoter; however, HMGA1 coexpression still suppressed promoter activity while HIPK2 restored it (Figure 3D), suggesting that the HIPK2 kinase activity required to revert the effect of HMGA1 is not confined to the Ser46 phosphorylation of p53.

HIPK2 is required for the HMGA1-mediated repression of $p 53$ induced apoptosis. To verify the requirement for HIPK2 in HMGA1-mediated repression of p53 apoptotic activity, expression of the endogenous HIPK2 protein was depleted by stable transfection of H1299 cells with a PSUPER vector carrying HIPK2- or $\beta$-gal-specific RNA-interfering sequences (see Methods). As shown in Figure 4A, HIPK2 expression was decreased in the H1299 HIPK2-interfered (HIPK2i) cells compared with that of control vector-transfected H1299 cells ( $\beta$-Gali). The 2 populations were infected with Adp53 in the presence or absence of overexpressed HMGA1. As expected from previous data (37), HIPK2-depleted cells were more resistant to p53mediated apoptosis (Figure 4B). However, when HMGA1 was overexpressed together with p53, the cells were protected from apoptosis only in the presence of endogenous HIPK2, while no protection was observed in the HIPK2-depleted cells (Figure 4B, upper panel). This protection was not caused by differences in the expression levels of the transduced proteins, since the amounts of p53 and HMGA1 were comparable in the 2 populations (Figure 4B, lower panel). To verify whether activation of the endogenous $\mathrm{p} 53$ protein can induce a similar outcome, WT p53-carrying RKO cells were stable transfected with the same pSUPER vectors described above, and HIPK2 depletion was verified by Western blotting (Figure 4C). Consistent with the results obtained by exogenous $\mathrm{p} 53$ expression, HMGA1 overexpression protected from UV light-induced apoptosis only in HIPK2-expressing cells (Figure 4D). These data indicate that HMGA1 represses the apoptotic activity of $\mathrm{p} 53$ by counteracting HIPK2.

HMGA1 exerts its antiapoptotic function by interfering with the nuclear localization of HIPK2. We previously showed that HMGA1 physically interacts with HIPK2 (35) and that HIPK2 colocalizes with p53 in the nuclear bodies and activates p53 apoptotic function 


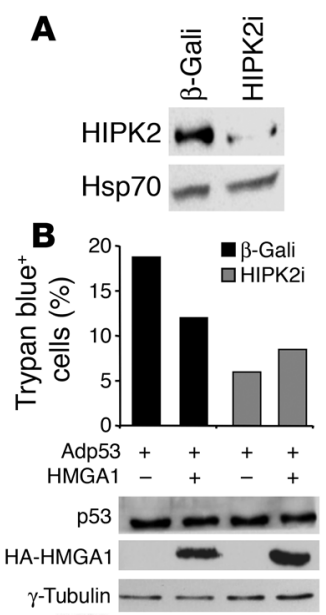

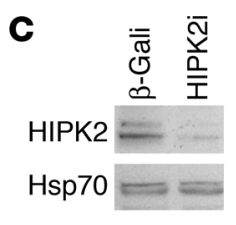

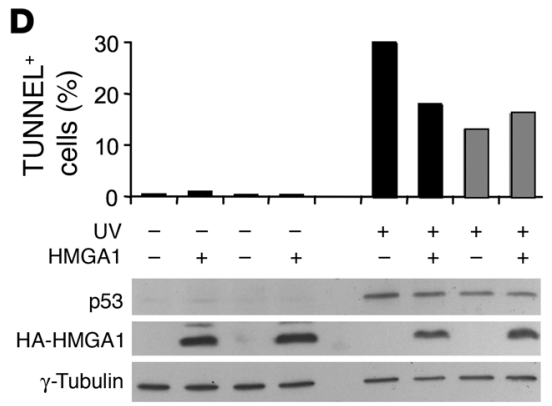

\section{Figure 4}

HIPK2 is required for HMGA-mediated repression of apoptosis. (A) Western blot analysis of endogenous HIPK2 in p53-null H1299 cells stably transfected with pSUPER-lacZ ( $\beta$-Gali) or pSUPERHIPK2 (HIPK2i) vectors. Heat shock protein 70 (Hsp70) was used as loading control. (B) The same cells used in $\mathbf{A}$ were infected with Adp53 and transfected with the pCEFL-Hmga1 vector or PCEFL empty vector. Cell death was measured as in Figure 2B, while TCEs from the same cells used in B were analyzed by Western blot as in Figure 2B for the indicated proteins. (C) Western blot analysis of endogenous HIPK2 in WT p53-expressing RKO cells stably transfected as in A. (D) The same cells used in $\mathbf{C}$ were transfected with HMGA1 and irradiated with UV light $\left(50 \mathrm{~J} / \mathrm{m}^{2}\right)$ to activate the endogenous p53. The apoptotic index was calculated by TUNEL assay.
$(29,30)$. Because HMGA1 inhibits p53-induced apoptosis by acting on HIPK2, we evaluated the interaction and the localization of HIPK2 in the presence or absence of overexpressed HMGA1. We confirmed the physical interaction between the 2 proteins (Figure $5 \mathrm{~A}$ ) and observed a dose-dependent cytoplasmic localization of HMGA1 (Figure 5, B and C) that was strongly associated with cytoplasmic relocalization of HIPK2 (Figure 5D). This subcellular distribution of HIPK2 depended on the relative ratio between HMGA1 and HIPK2, because HIPK2 overexpression restored HIPK2 in the nuclei (Figure 5, E and F). Consistently, specific antisense oligonucleotide depletion of HMGA1 on RKO cells, which endogenously express HIPK2, WT p53, and high levels of HMGA1 (Figure 6A), resulted in increased nuclear localization of endogenous HIPK2 protein, as assessed by Western blot analysis on separated nuclear/cytoplasmic cell extracts, while p53 remained in the nucleus in each condition (Figure 6B). Together with our observation that this specific antisense oligonucleotide treatment was also associated with increased UV light-induced apoptosis (Figure 2D and ref. 20), these results strongly suggest that HMGA1 inhibits p53 apoptotic function by interfering with the nuclear localization of the p53 activator HIPK2.

To confirm this hypothesis, enhanced GFP-HIPK2 (EGFPHIPK2) or its kinase-dead EGFP-K221R mutant were expressed in RKO cells in the presence or absence of the nuclear export inhibitor leptomycin B (LMB) (38). LMB treatment promoted more robust nuclear compartmentalization of HIPK2 (Figure 6C) and stronger induction of apoptosis than did mock treatment (Figure $6 \mathrm{D}$ ), supporting the notion that HIPK2 nuclear localization is necessary for p53 activation and function (39).

HMGA1 overexpression strongly associates with HIPK2 cytoplasmic localization in breast carcinomas. To evaluate whether the relationship between HMGA1 overexpression and HIPK2 subcellular localization defined in tumor cell lines can occur in vivo, we analyzed both HMGA1 expression and HIPK2 nuclear and cytoplasmic compartmentalization in 69 stage I and stage II breast cancer patients whose clinical characteristics have been described previously (40). As shown in Table 1, HMGA1 was overexpressed in 35 of 69 cases (51\%) while HIPK2 was positive in 44 tumors (64\%), with a cytoplasmic pattern of reactivity in 27 patients (39\%) and a nuclear pattern in 17 (25\%). Of interest, HMGA1 overexpression significantly correlated with the cytoplasmic compartmentalization of HIPK2 (i.e., in the HMGA1-positive samples, HIPK2 showed a cytoplasmic pattern in $57 \%$ of the cases and a nuclear one in $26 \%$; $P<0.0001$, $\chi^{2}$ test). In addition, in the HMGA1-positive samples, the percentage of p53-positive (surrogate of mutant p53) breast cancers was 55\% in patients presenting nuclear localization of HIPK 2 and $37 \%$ in those presenting a cytoplasmic one (Figure 7A). To evaluate whether this correlation was associated with resistance to apoptosis, as would be predicted by our in vitro model, we performed a TUNEL assay on 4 selected groups of HMGA1-positive breast carcinomas (Figure 7, B and C) according to their cytoplasmic (Figure 7D) or nuclear (Figure 7E) positivity for HIPK2 and their p53 status. As summarized in Figure 7F, when HIPK2 was relocalized in the cytoplasm, we observed a low apoptotic index in both p53-negative (surrogate of WT p53) and p53-positive (surrogate of mutant p53) tumors (p53negative, $2.79 \pm 0.253$; 553 -positive, $2.32 \pm 0.244$ ). In contrast, when HIPK2 was localized in the nucleus, the apoptotic index was low only in the mutant p53-positive tumors (p53-positive, $2.76 \pm 0.500$; p53-negative, $9.00 \pm 2.546$ ), as expected. These results indicate that an association among HMGA1 overexpression, HIPK2 cytoplasmic localization, and resistance to apoptosis even in the presence of WT p53 can occur in vivo, at least in breast carcinoma.

\section{Discussion}

Defects in apoptosis are thought to play a major role in tumorigenesis as well as in tumor response to anticancer treatments (23). Recently, we reported that HMGA1, whose aberrant expression is implicated in the process of carcinogenesis, binds to the $\mathrm{p} 53$ oncosuppressor and inhibits its apoptotic activity (20). At the molecular level, this inhibition is associated with increased transcription of the p53 inhibitor MDM2 and repression of the p53 effectors $B A X$ and $p 21^{W A F 1}(20)$. These observations were subsequently confirmed and extended to members of the p53 family by Frasca and coauthors (41). In the present study, we investigated the mechanism through which HMGA1 modifies p53 transcriptional activity and inhibits apoptosis. We found that HMGA1 repressed p53 apoptotic activity by promoting the cytoplasmic relocalization of the 553 proapoptotic activator HIPK2.

HIPK2 physically and functionally interacts with p53 and activates its apoptotic function by specifically phosphorylating human p53Ser46 and mouse p53Ser58 $(29,30,42)$. In particular, it was shown that p53Ser46 phosphorylation changes the affinity of 553 for different promoters with a shift from growth arrestrelated genes to apoptosis-related ones $(27,28)$. HIPK2 promotes 
A
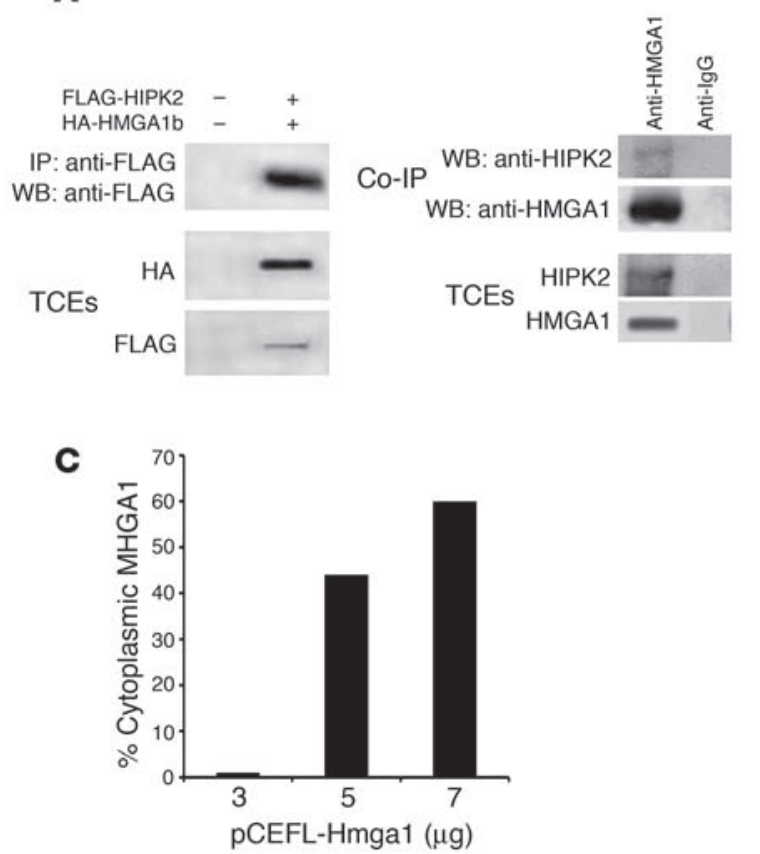

E

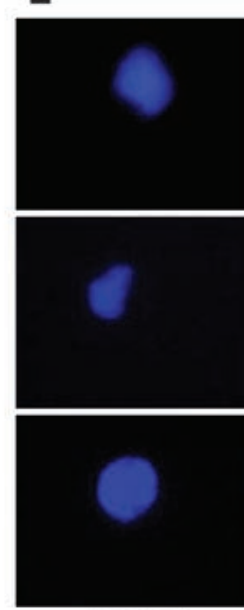

Hoescht
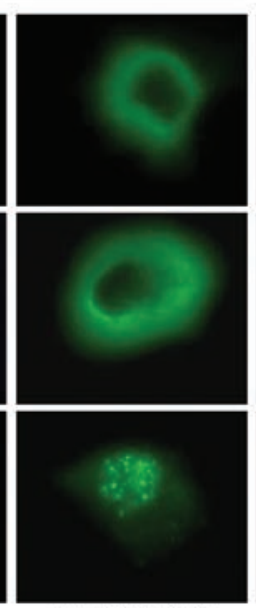

EGFP-HIPK2
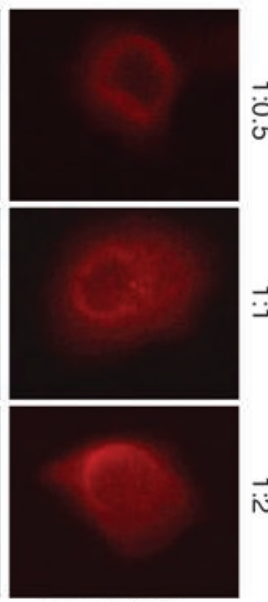

HA-HMGA1

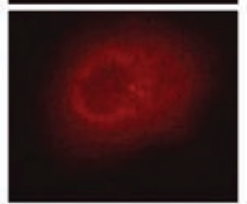

B
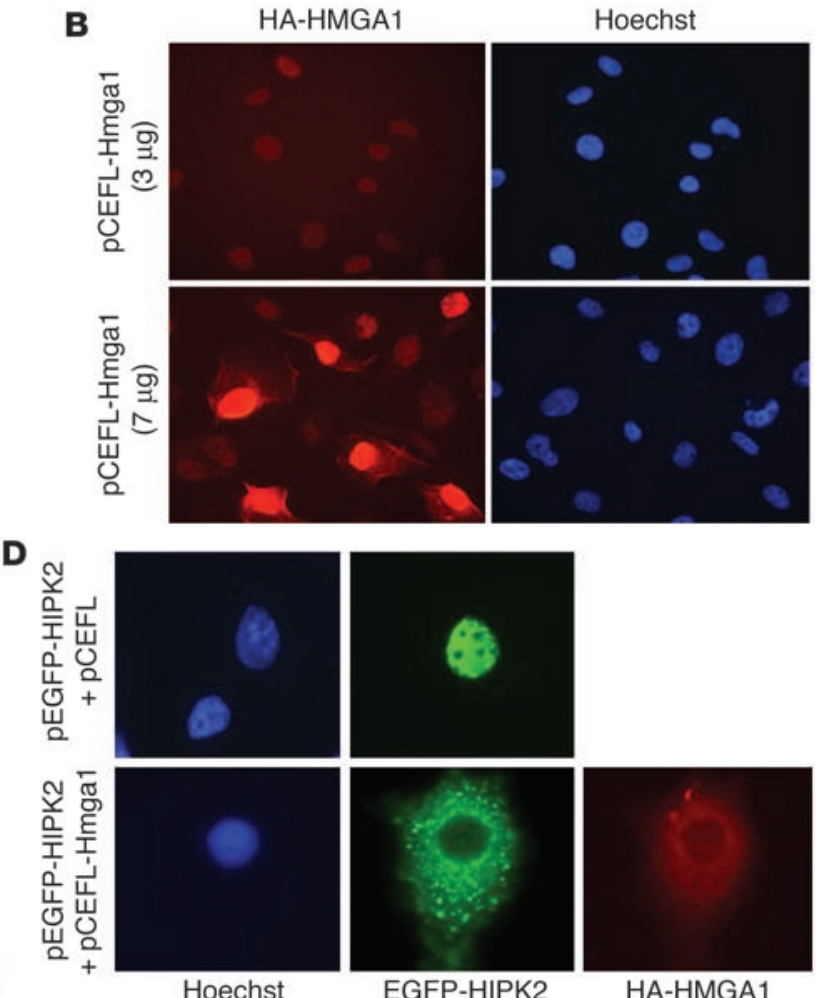

EGFP-HIPK2

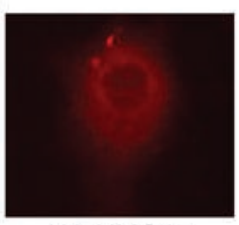

HA-HMGA1

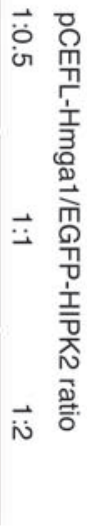

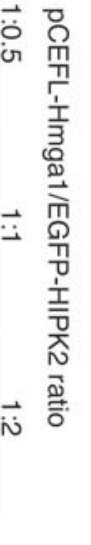

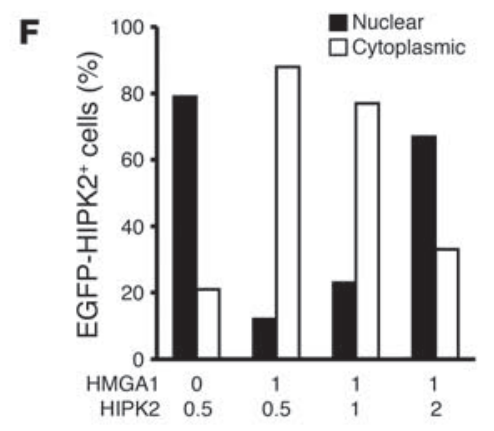

Figure 5

HMGA1 inhibits p53-induced apoptosis by interfering with HIPK2 nuclear localization. (A) For coimmunoprecipitation of exogenous proteins, $\mathrm{H} 1299$ cells were transfected as indicated and TCEs were immunoprecipitated with anti-HA Ab. Immunocomplexes were analyzed by Western blot (WB) as indicated. For coimmunoprecipitation of endogenous proteins, TCEs from H1299 cells were immunoprecipitated with anti-HMGA1 or anti-IgG as negative control and analyzed by Western blot. (B) H1299 cells were transfected with increasing doses of pCEFL-Hmga1. HA-HMGA1 expression was analyzed by immunofluorescence with anti-HA and tetramethylrhodamine isothiocyanate-conjugated Abs. Nuclei were stained with Hoechst. Images are from 1 representative experiment of the 3 performed. (C) H1299 cells were transfected and immunostained as in B; the percentage of HMGA1-positive cells with cytoplasmic staining was counted. (D) EGFP-HIPK2 subcellular localization in H1299 cells transfected with the indicated vectors. EGFP-HIPK2 is visible by its intrinsic green fluorescence. HA-HMGA1 expression was analyzed as in B. (E) H1299 cells were transfected with pCEFL-Hmga1 and pEGFP-HIPK2 expression vectors at the indicated molar ratios. EGFP-HIPK2 and HA-HMGA1 expression and their subcellular localization were analyzed by immunofluorescence as in D. (F) H1299 cells were transfected and immunostained as in E. The percentages of HMGA1-positive cells with nuclear or cytoplasmic staining of EGFP-HIPK2 were counted. Cells with a pCEFL-Hmga1 dose of 0 were cotransfected with pCEFL empty vector.

p53-mediated transcriptional activation of proapoptotic factors such as BAX, PIG3, Noxa, and p53-regulated apoptosisinducing protein and repression of antiapoptotic factor such as Galectin-3 through phosphorylation of human p53Ser46 and mouse p53Ser58 (27-30, 42, 43). HIPK2 and p53 colocalize in the nuclear bodies together with their typical components promyelocytic leukemia protein (PML) and SP100 nuclear antigen $(29,30,39,44)$. A PML-independent, nuclear, dotted distribution of EGFP-HIPK2 has also been reported; however, HIPK2dependent p53 phosphorylation and the subsequent p53-medi- 
A
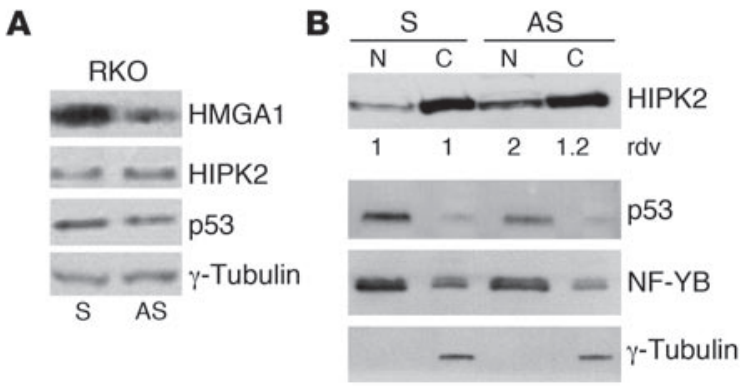

C
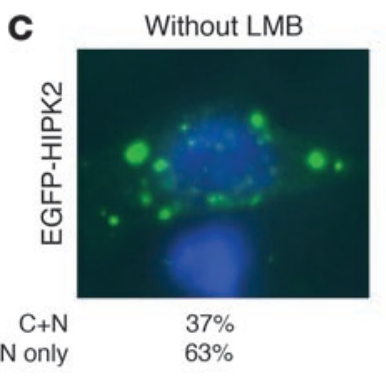

D

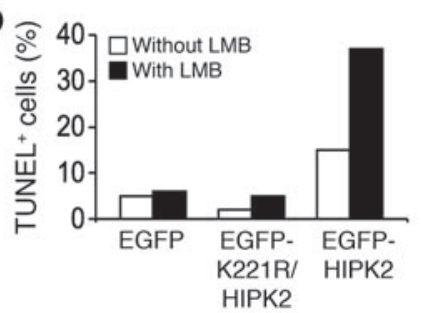

ated activity requires the presence of PML (39), indicating that HIPK2 nuclear localization is necessary for $\mathrm{p} 53$ activation. Here we show that the nuclear localization of HIPK2 was inhibited by HMGA1 overexpression and restored by HMGA1 depletion or HIPK2 overexpression. In addition, forced nuclear localization of HIPK2 induced by the nuclear export inhibitor LMB strongly increased HIPK2-induced apoptosis.

We previously showed that HMGA1 interacts with HIPK2. However, the functional relevance of this interaction was unclear (35). The data we describe here show that an imbalance in the ratio between HMGA1 and HIPK2 resulting in HMGA1 overexpression elicited an antiapoptotic effect by inducing HIPK2 accumulation in the cytoplasm and impairing p53Ser 46 phosphorylation. Conversely, overexpression of HIPK2 compared with that of HMGA1 reestablished HIPK2 nuclear localization and rescued the rate of p53-mediated apoptosis in the presence of HMGA1. This rescue was associated with recovery of $\mathrm{p} 53$ transcriptional activity on the $B A X, P I G 3$, and $p 21^{W A F I}$ promoters as well as further activation of the $m d m 2$ promoter.

Besides TP53 gene mutations, several mechanisms of $\mathrm{p} 53$ protein inactivation have been shown to contribute to tumor development, including p53 maintenance in the cytoplasm $(45,46)$. The finding that cytoplasmic localization of HIPK2 is determined by greater expression of HMGA1 than of HIPK2 strongly supports the idea that HMGA1 contributes to tumorigenicity by interfering with the tumor-suppressing activity of p 53 through cytoplasmic localization of its activator HIPK2. This mechanism might account for the progression of neoplasms in which p53 apoptotic activity is impaired, notwithstanding the absence of p53 gene mutations and/or deletions. It is noteworthy that an analysis of 69 breast carcinoma patients showed a significant associa-

\section{Figure 6}

HIPK2 nuclear localization strongly increases HIPK2-induced apoptosis. (A) Western blot analyses of endogenous HMGA1, HIPK2, and p53 in WT p53-carrying RKO cells transduced with HMGA1-specific sense or antisense oligonucleotides. $\gamma$-Tubulin was used as loading control. (B) From the same cells as in A, nuclear (N) and cytoplasmic (C) extracts were prepared 36 hours after transduction and analyzed by Western blotting for the indicated proteins. NF-YB (48) and $\gamma$-tubulin were used as markers of nuclear/cytoplasmic separation as well as loading controls. Quantitative analysis was performed, and the relative density value (rdv) of HIPK2 was calculated as a ratio between HIPK2 and NF-YB in the nuclear extracts and between HIPK2 and $\gamma$-tubulin in the cytoplasmic extracts. (C) RKO cells were transfected with EGFP-HIPK2 vector and cultured in the presence or absence of $10 \mathrm{nM}$ LMB for 16 hours. Subcellular localization of the EGFP construct was examined by fluorescence microscopy. Cells were simultaneously stained for DNA using Hoechst. The staining phenotype was categorized in 2 groups, 1 with nuclear HIPK2 staining only, and 1 with both nuclear and cytoplasmic staining. (D) RKO cells were transfected with the indicated EGFP vectors and treated with LMB as in C. At 20 hours after transfection, both floating and adherent cells were harvested to measure cell death by TUNEL assay. tion among HMGA1 overexpression, cytoplasmic localization of HIPK2, and low spontaneous apoptosis index (comparable to that observed and described in tumors carrying mutant p53), supporting the hypothesis that in these tumors there is an inactivation of p53 apoptotic function mediated by HMGA1-dependent cytoplasmic localization of HIPK2.

In conclusion, our data strongly support the existence of what we believe to be a new mechanism of p53 inactivation through HMGA1-mediated cytoplasmic localization of the p53 activator HIPK2. This mechanism might have important implications in tumorigenicity as well as in the development of tumor resistance to antineoplastic treatments.

\section{Methods}

Cell culture, transfections, and transactivation assays. HCT116, RKO, and H1299 cells were maintained in DMEM supplemented with $10 \%$ fetal calf serum (GIBCO; Invitrogen), glutamine, and antibiotics. Cells were transfected with plasmids by lipofectamine-plus reagent or with oligonucleotides by oligofectamine reagent (Invitrogen) as suggested by the manufacturer. Cells were transiently transfected with previously described reporter vectors $(20,29)$ and normalized with the use of a cotransfected $\beta$-gal construct. Luciferase activity was analyzed by Dual-Light System (Applied Biosystems).

For inhibition of HMGA1 expression, antisense and corresponding sense oligonucleotides were designed and synthesized specifically by BIOGNOSTIK. Oligonucleotides were added once at a concentration of $2 \mathrm{mM}$. For inhibition of nuclear exports, cells were treated with $1 \mathrm{nM}$ LMB for 18 hours.

Expression constructs. The pCAG-p53, pCAG-p53Ser46, pCMV-Hmga1, pCEFL-HA-HMGA1, pFLAG-HIPK2, pFLAG-K221R/HIPK2, pEGFP-HIPK2, and pEGFP-K221R constructs have been described previously $(29,35,42)$. 


\begin{tabular}{|c|c|c|c|}
\hline HIPK2 expression & HMGA1-positive & HMGA1-negative & Total cases \\
\hline Nuclear & $11(26 \%)$ & $6(22 \%)$ & $17(25 \%)$ \\
\hline Cytoplasmic & $24(57 \%)$ & $3(11 \%)$ & 27 (39\%) \\
\hline Negative & $7(17 \%)$ & $18(67 \%)$ & $25(36 \%)$ \\
\hline Total cases & 42 & 27 & 69 \\
\hline
\end{tabular}

$P<0.0001, \chi^{2}$ test; $P<0.005, \chi^{2}$ test for trend. The latter test indicates how much of the association between HMGA1 and HIPK2 nuclear or cytoplasmic localization is accounted for by linear trend.

Western blotting and coimmunoprecipitation. Total cell extracts (TCEs) were prepared with lysis buffer (50 mM Tris HCl, pH 7.5; 5 mM EDTA; $300 \mathrm{mM}$ $\mathrm{NaCl} ; 150 \mathrm{mM} \mathrm{KCl} ; 1 \mathrm{mM}$ dithiothreitol; $1 \%$ Nonidet P40; and a mix of protease inhibitors) or in nondenaturing buffer $(50 \mathrm{mM}$ Tris- $\mathrm{HCl}, \mathrm{pH} 7.5$; $150 \mathrm{mM} \mathrm{NaCl}$; $0.5 \%$ Triton; and $5 \mathrm{mM}$ EDTA] for coimmunoprecipitation. Immunoprecipitation was carried out by incubating 1-3 mg of TCEs with Abs preadsorbed to protein G-agarose (Pierce Biotechnology). Immunocomplexes were collected by centrifugation, separated by SDS-PAGE, and blotted onto nitrocellulose membrane (Bio-Rad). Differential nuclear and cytoplasmic cell lysates were obtained as reported previously (47). Protein extracts were separated by SDS-PAGE and transferred to Immobilon-P Transfer membranes (Millipore). Membranes were blocked with 5\% nonfat milk proteins and incubated with Abs at the appropriate dilutions. The filters were incubated with horseradish peroxidase-conjugated secondary Abs, and the signals were detected with ECL (Amersham). The Abs used for Western blotting were as follows: anti-FLAG M5 $\mathrm{mAb}$ (Sigma-Aldrich); anti-HA 12CA5 mAb (Roche); anti-p53 DO1 mAb and anti-heat shock protein 70 polyclonal Ab (Santa Cruz Biotechnology Inc.); sheep anti-p53 polyclonal Ab (Ab-7; Calbiochem); rabbit anti-phosphorylated p53Ser46 polyclonal and rabbit anti-phosphorylated p53Ser15 polyclonal Abs (Cell Signaling Technology); anti-nuclear factor YB (anti-NF-YB) (48); anti-HMGA1 polyclonal Ab (raised against a synthetic peptide located in the NH2-terminal region; ref. 20); and anti-HIPK2 polyclonal $\mathrm{Ab}$ (30). Anti- $\gamma$-tubulin (Santa Cruz Biotechnology Inc.) was used for loading control.

Recombinant adenoviruses and adenoviral infection. The early genes 1 and 3-defective recombinant adenovirus dl70.3, Adp53 (49), and AdGFP and AdHMGA1 (19) were amplified and titrated on HEK293 cells as described previously (49).

Cell viability and TUNEL assays. Both floating and adherent cells were collected and counted in a hemocytometer after the addition of trypan blue. The percentage of dead cells (e.g., number of blue cells per total number of cells) was determined by scoring 100 cells per chamber 3 times. Cell numbers were determined in duplicate.

For TUNEL assay on in vitro cell lines, both floating and adherent cells were spun onto slides by cytocentrifugation. After fixing in $4 \%$ formaldehyde in PBS, cells were incubated with fluorescein-conjugated dUTP terminal deoxynucleotide transferase mixture for TUNEL reaction (Roche) according to the manufacturer's instructions, counterstained with $1 \mathrm{mg} / \mathrm{ml}$ Hoechst 33258 for 2 minutes, and mounted with coverslip in $25 \%$ glycerol in PBS. At least 400 cells were counted in each plate.

For TUNEL assay on human breast cancer tissue, the fluorescent in situ detection of apoptosis in formalin-fixed, paraffin-embedded breast cancer tissues was performed using the MEBSTAIN Apoptosis kit II (IMMUNO$\mathrm{TECH}$ ) according to the manufacturer's instructions. After deparaffinization and rehydration, $50 \mu \mathrm{l}$ of terminal deoxynucleotidyl transferase-mediated nick end-labeling reaction mixture was applied to slides, which were then incubated at $37^{\circ} \mathrm{C}$ for 60 minutes. Sections were examined using a fluorescence microscope, and the number of apoptotic cells was counted in 8 high-power fields (original magnification, $\times 400$ ) per section. Counts were averaged to determine the number of apoptotic cells.

Indirect immunofluorescence. Cells plated in $35-\mathrm{mm}$ dishes were fixed in $2 \%$ formaldehyde in PBS and permeabilized in a solution of $0.25 \%$ Triton $\mathrm{X}-100$ in PBS. Immunofluorescence was obtained with the anti-HA 12CA5 $\mathrm{mAb}$ (Roche) and the tetramethylrhodamine isothiocyanate-conjugated goat anti-mouse IgG (Jackson ImmunoResearch Laboratories Inc.). Cells were stained simultaneously for DNA with Hoechst 33342 before observation with a fluorescent microscope (Zeiss).

Plasmid construction for RNA interference and cell transfection. The PSUPER- $\beta$-gal and pSUPER-HIPK2 plasmids were constructed as reported previously (43), and Western blot analysis for STAT1 protein was used to exclude stimulation of IFN production in the stably transfected cell lines. The pSUPER- $\beta$-gal vector carrying an interfering sequence for the $L a c Z$ gene was used as a control.

Patients and tissue specimens. The 69 stage I, stage IIa, and stage IIb breast cancer patients (median age, 50 years; range, $30-76$ years) included in this
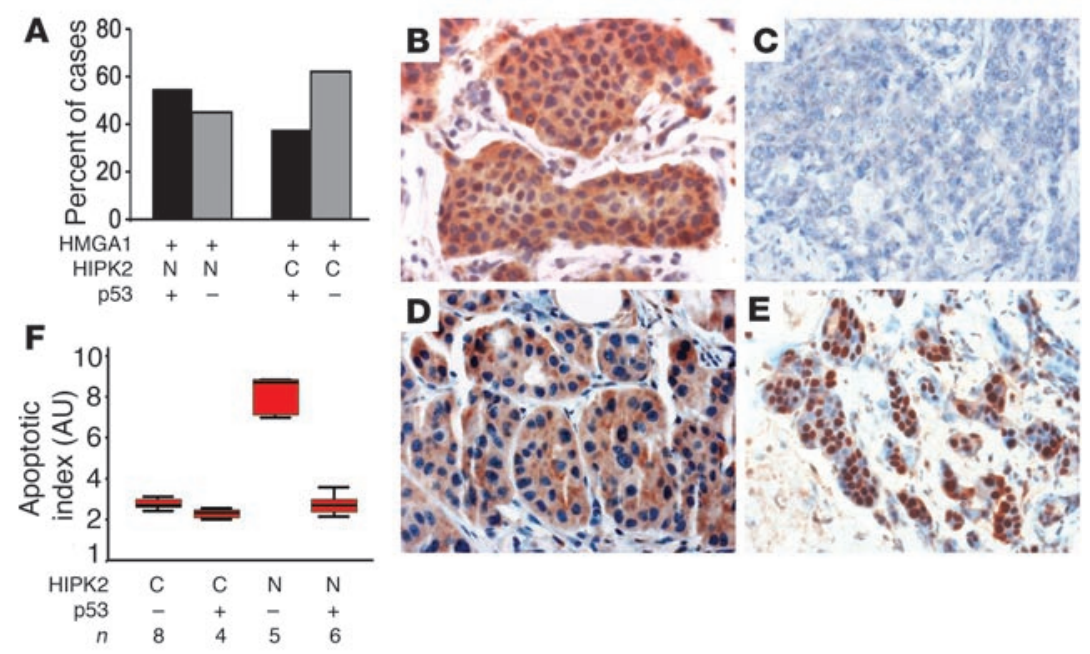

Figure 7

HMGA1 and HIPK2 immunostaining in breast cancer. (A) Histogram showing the distribution of p53 immunostaining in 44 tumor samples that expressed both HMGA1 and HIPK2. (B-E) Streptavidin-biotin immunoperoxidase staining of invasive breast ductal carcinomas displaying HMGA1 overexpression (B), HMGA1 absence (C), and distinct cytoplasmic (D) or nuclear (E) localization of HIPK2. Original magnification, $\times 40$. (F) Quantification of apoptotic index in 4 groups of HMGA1 positive breast carcinomas according to their HIPK2/p53 phenotypes ( 8 cases: HIPK2 positive in the cytoplasm/p53 negative; 4 cases: HIPK2 positive in the cytoplasm/p53 positive; 5 cases: HIPK2 positive in the nucleus/p53 negative, and 6 cases: HIPK2 positive in the nucleus/p53 positive). The apoptotic index was counted in 10 fields per tumor evaluating the apoptotic index mean value in each group. The mean apoptotic index in HIPK2 nuclear-positive, p53-negative tumors was significantly higher than those of the other 3 groups $(P<0.0001$; Bonferroni test). Lines represent median values, shaded boxes represent twenty-fifth and seventy-fifth percentiles, and whiskers represent minimum and maximum values. $Y$ axis values indicate the mean value of the number of cells. Data are mean \pm SD. 
study were surgically treated at the Regina Elena Cancer Institute (40). This series included 58 invasive ductal carcinomas, 7 invasive lobular carcinomas, 2 tubular carcinomas, and 2 medullary carcinomas, of which 51 were $\mathrm{T} 1$ and $18 \mathrm{~T} 2$ and 50 were node-negative and 19 node-positive. Tumors were staged according to the Unione Internationale Contre le Cancer TNM system 2002, and graded according to Bloom and Richardson (50). The study was reviewed and approved by the ethics committee of the Regina Elena National Cancer Institute, and written informed consent was obtained from all patients.

Immunohistochemistry. Breast cancer specimens were fixed for 18-24 hours in $4 \%$ buffered formaldehyde and then processed through to paraffin wax. HMGA1, HIPK2, and p53 were evaluated by immunohistochemistry on 5 - $\mu \mathrm{m}$-thick paraffin-embedded tissues. Sections harvested on SuperFrost Plus slides (Menzel-Glaser) were deparaffinized, rehydrated, and pretreated in a thermostatic bath at $96^{\circ} \mathrm{C}$ for 40 minutes in $10 \mathrm{mM}$ citrate buffer ( $\mathrm{pH}$ 6). Polyclonal Abs directed against HMGA1 (diluted 1:50) and HIPK2 $(1 \mu \mathrm{g} / \mathrm{ml})$ and anti-p53 mAb purchased from DakoCytomation (clone DO7) were incubated for 60 minutes at room temperature. The reactions were revealed using Super Sensitive Link-Label Detection System purchased from Biogenex (Space), using 3-amino-9-ethylcarbazole (AEC substrate chromogen; DakoCytomation) as chromogenic substrate. All sections were slightly counterstained with Mayer hematoxylin and mounted in aqueous mounting medium (Glycergel; DakoCytomation). HMGA1 and HIPK2 proteins were considered overexpressed when more than $10 \%$ of the neoplastic cells presented a strong immunoreaction in the cytoplasm and/or in the nucleus. p53 was considered positive only when a distinct nuclear stain was observed in more than $10 \%$ of cancer cells. Evaluation of the immunohistochemical data was performed independently and in blinded manner by 2 investigators (M. Mottolese and A.D. Benedetto).

Statistics. The $\chi^{2}$ test was used to test the relationship between HMGA1 overexpression and HIPK2 nuclear or cytoplasmic compartmentalization. A $P$ value of less than 0.05 was considered statistically significant for both tests. The tests indicate how much of the association between HMGA1 and
HIPK2 nuclear or cytoplasmic localization is accounted for by linear trend. To determine differences in apoptotic index among tumors according to HMGA1, HIPK2, and p53 immunohistochemical phenotypes, the Bonferroni test was used.

\section{Acknowledgments}

We are grateful to Laura Ciuffini and Francesca Puglisi for sharing their experiments on subcellular fractionations and to M. Lienhard Schmitz for the kind gift of the anti-HIPK2 polyclonal Ab. This work was supported by grants from Associazione Italiana per la Ricerca sul Cancro, the Progetto Finalizzato "Biotecnologie" of the CNR, and MURST projects "Terapie antineoplastiche innovative" and "Piani di Potenziamento della Rete Scientifica e Tecnologica” from the Ministero della Salute as well as by EC FP6 funding (contract 503576). This publication reflects the authors' views and not necessarily those of the European Union. The EU is not liable for any use that may be made of the information contained herein. We thank the Associazione Partenopea per le Ricerche Oncologiche (APRO) for its support. Cinzia Renaldo is recipient of a FIRC fellowship.

Received for publication July 25, 2006, and accepted in revised form December 5, 2006.

Address correspondence to: Silvia Soddu, Dipartimento di Oncologia Sperimentale, Istituto Nazionale dei Tumori Regina Elena, via delle Messi d'Oro, 156, 00158 Rome, Italy. Phone: 39-0652662563; Fax: 39-065266-2505; E-mail: soddu@ifo.it.

Giovanna Maria Pierantoni and Cinzia Rinaldo contributed equally to this work.

Silvia Soddu and Alfredo Fusco are co-senior authors.
1. Grosschedl, R., Giese, K., and Pagel, J. 1994. HMG domain proteins: architectural elements in the assembly of nucleoprotein structures. Trends Genet. 10:94-100.

2. Reeves, R., and Nissen, M.S. 1990. The A-T-DNAbinding domain of mammalian high mobility group I chromosomal proteins. A novel peptide motif for recognizing DNA structure. J. Biol. Chem. 265:8573-8582.

3. Reeves, R. 2000. Structure and function of the HMGI(Y) family of architectural transcription factors. Environ. Health Perspect. 108:803-809.

4. Thanos, D., and Maniatis, T. 1995. Virus induction of human IFN beta gene expression requires the assembly of an enhanceosome. Cell. 83:1091-1100.

5. Zhou, X., Benson, K.F., Ashar, H.R., and Chada, K. 1995. Mutation responsible for the mouse pygmy phenotype in the developmentally regulated factor HMGI-C. Nature. 376:771-774.

6. Chiappetta, G., et al. 1996. High level expression of the HMGA1 gene during embryonic development. Oncogene. 13:2439-2446.

7. Chiappetta, G., et al. 1995. The expression of the high mobility group HMGA1 proteins correlates with the malignant phenotype of human thyroid neoplasms. Oncogene. 10:1307-1314.

8. Chiappetta, G., et al. 1998. Detection of high mobility group I HMGI(Y) protein in the diagnosis of thyroid tumors: HMGI(Y) expression represents a potential diagnostic indicator of carcinoma. Cancer Res. 58:4193-4198.

9. Fedele, M., et al. 1996. Human colorectal carcinomas express high levels of high mobility group HMGI(Y) proteins. Cancer Res. 56:1896-1901.
10. Abe, N., et al. 1999. Determination of high mobility group $\mathrm{I}(\mathrm{Y})$ expression level in colorectal neoplasias: a potential diagnostic marker. Cancer Res. 59:1169-1174.

11. Chiappetta, G., et al. 2001. High mobility group HMGI(Y) protein expression in human colorectal hyperplastic and neoplastic diseases. Int. J. Cancer. 91:147-151.

12. Tamimi, Y., et al. 1993. Increased expression of high mobility group protein $\mathrm{I}(\mathrm{Y})$ in high grade prostatic cancer determined by in situ hybridization. Cancer Res. 53:5512-5516.

13. Abe, N., et al. 2002. Diagnostic significance of high mobility group $\mathrm{I}(\mathrm{Y})$ protein expression in intraductal papillary mucinous tumors of the pancreas. Pancreas. 25:198-204.

14. Bandiera, A., et al. 1998. Expression of HMGI(Y) proteins in squamous intraepithelial and invasive lesions of the uterine cervix. Cancer Res. 58:426-431.

15. Masciullo, V., et al. 2003. HMGA1 protein overexpression is a frequent feature of epithelial ovarian carcinomas. Carcinogenesis. 24:1191-1198.

16. Chiappetta, G., et al. 2004. HMGA1 protein overexpression in human breast carcinomas: correlation with ErbB2 expression. Clin. Cancer Res. 10:7637-7644.

17. Berlingieri, M.T., et al. 1995. Inhibition of HMGI-C protein synthesis suppresses retrovirally induced neoplastic transformation of rat thyroid cells. Mol. Cell. Biol. 15:1545-1553.

18. Berlingieri, M.T., Pierantoni, G.M., Giancotti, V., Santoro, M., and Fusco, A. 2002. Thyroid cell transformation requires the expression of the HMGA1 proteins. Oncogene. 21:2971-2980.
19. Scala, S., Portella, G., Fedele, M., Chiappetta, G., and Fusco, A. 2000. Adenovirus-mediated suppression of HMGI(Y) protein synthesis as potential therapy of human malignant neoplasias. Proc. Natl. Acad. Sci. U. S. A. 97:4256-4261.

20. Pierantoni, G.M., et al. 2006. High Mobility Group A1 (HMGA1) proteins interact with p53 and interfere with its apoptotic function. Cell Death Differ. 13:1554-1563.

21. Vousden, K.H. 2000. p53: death star. Cell. 103:691-694.

22. Vogelstein, B., Lane, D., and Levine, A.J. 2000. Surfing the p53 network. Nature. 408:307-310.

23. Schmitt, C.A., et al. 2002. Dissecting p53 tumor suppressor functions in vivo. Cancer Cell. 1:289-296.

24. Appella, E., and Anderson, C.W. 2000. Signaling to p53: breaking the post-translational modification code. Pathol. Biol. 48:227-245.

25. Meek, J. 2004. The p53 response to DNA damage. DNA Repair. 3:1049-1056.

26. Bulavin, D.V., et al. 1999. Phosphorylation of human $\mathrm{p} 53$ by $\mathrm{p} 38$ kinase coordinates $\mathrm{N}$-terminal phosphorylation and apoptosis in response to UV radiation. EMBO J. 18:6845-6854.

27. Oda, K., et al. 2000. p53AIP1, a potential mediator of $\mathrm{p} 53$-dependent apoptosis, and its regulation by Ser-46-phosphorylated p53. Cell. 102:849-862.

28. Mayo, L.D., et al. 2005. Phosphorylation of human p53 at serine 46 determines promoter selection and whether apoptosis is attenuated or amplified. J. Biol. Chem. 280:25953-25959.

29. D’Orazi, G., et al. 2002. Homeodomain-interacting protein kinase-2 phosphorylates p53 at Ser 46 and mediates apoptosis. Nat. Cell Biol. 4:11-19. 
30. Hofmann, T.G., et al. 2002. Regulation of p53 activity by its interaction with homeodomain-interacting protein kinase-2. Nat. Cell Biol. 4:1-10.

31. Kim, E.-J., Park, J.-S., and Um, S.-J. 2002. Identification and characterization of HIPK2 interacting with p73 and modulating functions of the p53 family in vivo. J. Biol. Chem. 277:32020-32028.

32. Zhang, Q., Yoshimatsu, Y., Hildebrand, J., Frisch, S.M., and Goodman, R.H. 2003. Homeodomain interacting protein kinase 2 promotes apoptosis by down-regulating the transcriptional co-repressor CtBP. Cell. 115:177-186.

33. Wang, Y., Debatin, K.M., and Hug, H. 2001. HIPK2 overexpression leads to stabilization of p53 protein and increased p53 transcriptional activity by decreasing Mdm2 protein levels. BMC Mol. Biol. 2:8-16.

34. Rui, Y., et al. 2004. Axin stimulates p53 functions by activation of HIPK2 kinase through multimeric complex formation. EMBO J. 23:4583-4594.

35. Pierantoni, G.M., et al. 2001. High mobility group I (Y) proteins bind HIPK2, a serine-threonine kinase protein which inhibits cell growth. Oncogene. 20:6132-6141.

36. Di Stefano, V., Rinaldo, C., Sacchi, A., Soddu, S. and D'Orazi, G. 2004. Homeodomain-interacting protein kinase- 2 activity and $\mathrm{p} 53$ phosphorylation are critical events for cisplatin-mediated apoptosis.
Exp. Cell Res. 293:311-320

37. Di Stefano, V., Soddu, S., Sacchi, A., and D'Orazi, G. 2005. HIPK2 contributes to PCAF-mediated p53 acetylation and selective transactivation of p21(Waf1) after nonapoptotic DNA damage. Oncogene. 24:5431-5442.

38. Fukuda, M., et al. 1997. CRM1 is responsible for intracellular transport mediated by the nuclear export signal. Nature. 390:308-311.

39. Moller, A., et al. 2003. PML is required for homeodomain-interacting protein kinase 2 (HIPK2)mediated p53 phosphorylation and cell cycle arrest but is dispensable for the formation of HIPK domains. Cancer Res. 63:4310-4314.

40. Mottolese, M., et al. 2007. Bio-pathologic characteristics according to chromosome 11 aneusomy and cyclin D1 gene status in surgically resected stage I and II breast cancer: identification of an adverse prognostic profile. Am. J. Surg. Pathol. In press.

41. Frasca, F., et al. 2006. HMGA1 inhibits the function of p53 family members in thyroid cancer cells. Cancer Res. 66:2980-2989.

42. Cecchinelli, B., et al. 2006. Ser58 of mouse p53 is the homologue of human Ser46 and is phosphorylated by HIPK2 in apoptosis. Cell Death Differ. 13:1994-1997.

43. Cecchinelli, B., et al. 2006. Repression of the antiapoptotic molecule galectin-3 by HIPK2-activated p53 is required for p53-induced apoptosis. Mol. Cell. Biol. 26:4746-4757.

44. Gostissa, M., Hofmann, T.G., Will, H., and Del Sal, G. 2003. Regulation of p53 functions: let's meet at the nuclear bodies. Curr. Opin. Cell Biol. 15:351-357. 45. Ostermeyer, A.G., Runko, E., Winkfield, B., Ahn, B., and Moll, U.M. 1996. Cytoplasmically sequestered wild-type p53 protein in neuroblastoma is relocated to the nucleus by a C-terminal peptide. Proc. Natl. Acad. Sci. U. S. A. 93:15190-15194.

46. Lu, W., et al. 2000. Nuclear exclusion of p53 in a subset of tumors requires MDM2 function. Oncogene. 19:232-240.

47. Schreiber, E., Matthias, P., Muller, M.M., and Schaffner, W. 1989. Rapid detection of octamer binding proteins with mini-extracts, prepared from a small number of cells. Nucleic Acid Res. 17:6419-6420.

48. Frontini, M., Imbriano, C., Manni, I., and Mantovani, R. 2004. Cell cycle regulation of NF-YC nuclear localization. Cell Cycle. 3:217-222.

49. Bacchetti, S., and Graham, F. 1993. Inhibition of cell proliferation by an adenovirus vector expressing the human wild type p53 protein. Int. J. Oncol. 3:781-788.

50. International Union Against Cancer. 2002. TNM classification of malignant tumors. L.H. Sobin and Ch. Witteking, editors. Wilery-Liss. New York, New York, USA. 272 pp. 